\title{
AVALIAÇÃO DOS EFEITOS DO TREINAMENTO AERÓBIO INTRADIALÍTICO EM PACIENTES RENAIS CRÔNICOS
}

\author{
EFFECTS OF INTRADIALYTIC AEROBIC TRAINING IN PATIENTS WITH CHRONIC RENAL FAILURE
}

\author{
Filipe Fernandes Oliveira Dantas ${ }^{\mathrm{a}^{*}}$ e Natália Maria Conceição Figueirôa ${ }^{\mathrm{b} *}$ \\ afilipepersonal@hotmail.com, bnatyfigueiroa@yahoo.com.br \\ *Centro Universitário do Rio Grande do Norte
}

Data de entrada do artigo: 05/12/2013

Data de aceite do artigo: 02/06/2014

\section{RESUMO}

Introduçáo: A doença renal crônica é atualmente um dos principais problemas de saúde no mundo. Como forma de tratamento dos pacientes renais crônicos, a terapia renal substitutiva, conhecida como hemodiálise, se faz necessária. Recentemente, vem sendo discutida na literatura a utilização de programas de exercícios físicos, durante a sessão de hemodiálise (intradialítico). Objetivo: Analisar a influência do treinamento aeróbio intradialítico em parâmetros fisiológicos, bioquímicos e funcionais, nos pacientes renais crônicos. Materiais e métodos: Este ensaio clínico controlado e randomizado consistiu num grupo experimental (com exercício) e um grupo controle. Os pacientes do grupo experimental fizeram 12 semanas de exercícios realizados num cicloergômetro, três dias por semana, numa intensidade correspondente a $50 \%$ - 60\% da frequência cardíaca de reserva. Foram feitas avaliações pré e pós-intervenção, tanto em variáveis fisiológicas (pressão arterial sistólica, pressão arterial diastólica e frequência cardíaca de repouso) quanto em variáveis bioquímicas (níveis séricos de ureia, creatinina, hemácias e hematócrito). Adicionalmente foi avaliada a capacidade funcional dos pacientes. Aplicaram-se os testes estatísticos Wilcoxon e U de MannWhitney, respectivamente, para as avaliaçóes intragrupo e intergrupo, sempre considerando um nível de significância menor do que 5\%. Resultados: Foi encontrado no grupo experimental melhora significativa dos valores de pressão arterial sistólica $(p=0,044)$, assim como redução significativa nos níveis séricos de ureia $(p=0,008)$. Além disso, constatou-se melhora significativa da capacidade funcional dos pacientes $(p=0,0001)$. Conclusáo: $\mathrm{O}$ treinamento aeróbio intradialítico foi capaz de proporcionar melhoras em variáveis fisiológicas, bioquímicas e funcionais nos pacientes submetidos à hemodiálise.

Palavras-chave: Exercício; terapia de substituição renal; pressão arterial; ureia.

\section{ABSTRACT}

Introduction: Chronic kidney disease is currently one of the major health problems in the world. A treatment of patients with chronic renal failure, renal replacement therapy, known as hemodialysis, is required. Recently, it has been discussed in the literature the use of physical exercise programs during the hemodialysis session (intradialytic). Objective: To analyze the influence of intradialytic aerobic training on physiological, biochemical and functional parameters, in patients with chronic kidney disease. Materials and Methods: This randomized controlled trial consisted of an experimental group (with exercise) and a control group. Patients in the experimental group did 12 weeks of exercises performed on a cycle ergometer, three days a week, at an intensity corresponding to $50 \%-60 \%$ of heart rate reserve. Assessments were made pre-and post-intervention both on physiological variables (systolic blood pressure, 
diastolic blood pressure and resting heart rate), as well as on biochemical variables (serum urea, creatinine, hemoglobin and hematocrit). We also evaluated the functional capacity of patients. We applied Wilcoxon and Mann-Whitney statistical tests, respectively, to evaluate intra-group and inter-group, always considering a significance level of less than $5 \%$. Results: We found a significant improvement in the experimental values of systolic blood pressure $(p=0,044)$, as well as significant reduction in serum urea $(p=0,008)$. Furthermore, there was a significant improvement in the functional capacity of patients $(p=0,0001)$. Conclusion: Intradialytic aerobic training was able to provide improvements in physiological, biochemical and functional parameters in patients undergoing hemodialysis.

Keywords: Exercise; renal replacement therapy; arterial pressure; urea.

\section{Introdução}

A doença renal crônica (DRC) atinge os rins, órgãos essenciais à manutenção da homeostase do corpo humano. Tal doença consiste na perda progressiva e irreversível da função deste órgão, fazendo com que os rins, além de não exercerem suas funções endócrinas normais, não consigam manter a homeostasia. Dessa forma, o indivíduo com DRC é acometido por um comprometimento de todo organismo ${ }^{1}$.

Há algumas situaçóes que lesam o rim agudamente, outras levam anos para o dano tornar-se aparente. As doenças que lesam as diferentes estruturas dos rins sáo, entre outras, as nefrites, o diabetes mellitus, a hipertensão arterial, infecçóes urinárias, obstruçóes das vias urinárias e histórico familiar de insuficiência renal crônica $(\text { IRC) })^{2}$.

Segundo Parsons et al. ${ }^{3}$, hemodiálise (HD) consiste numa terapia substitutiva para pacientes com DRC em seu estágio final. O processo ocorre por um método usado para filtrar o sangue das pessoas que possuem alguma doença no rim, em que este não é mais capaz de exercer essa função de filtragem. Os pacientes passam, em média, 40 horas mensais, durante vários anos, na unidade de HD ligado a uma máquina e monitorado por profissionais de saúde .

Há aproximadamente 30 anos, vem sendo discutida na literatura a utilização de programas de exercícios físicos, visando reabilitação física e funcional de indiví-

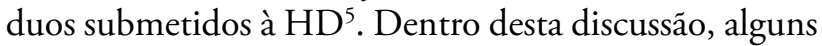
autores observaram que a utilização de um programa de exercícios interdialíticos (entre as sessóes de hemodiálise) geraram melhores resultados na aptidão aeróbia dos pacientes, entretanto, essa estratégia teve uma menor aderência quando comparada ao programa de exercício intradialítico (dentro da sessão de hemodiálise) ${ }^{6}$. Em função disso, tem sido defendida a utilização de exercícios físicos dentro da sessão de hemodiálise, inclusive pelo fato deste tipo de estratégia proporcionar melhorias em alguns marcadores de eficiência da hemodiálise, tais como o Kt/V, URR, CRR e o potássio plasmático ${ }^{7}$.

No entanto, apesar da observação de que a prática de exercício físico reduz a morbimortalidade em pacientes renais crônicos ${ }^{8}$, ainda não está bem definido se o exercício intradialítico pode proporcionar benefícios em alguns marcadores fisiológicos e funcionais, os quais determinam uma melhoria dos danos causados pela doença renal crônica.

Sendo assim, este estudo teve como objetivo analisar a influência do treinamento aeróbio intradialítico em parâmetros fisiológicos, bioquímicos e funcionais, nos pacientes renais crônicos da clínica de doenças renais, no município de Natal, RN.

\section{Materiais e métodos}

Este estudo foi caracterizado como um ensaio clínico controlado e randomizado, no qual os pacientes incluídos no estudo foram aleatoriamente distribuídos nos grupos experimentais e controle. A presente pesquisa foi aprovada pelo comitê de ética da Universidade Federal do Rio Grande do Norte. Os participantes foram esclarecidos a respeito de todo o protocolo da pesquisa e, posteriormente, assinaram o Termo de Consentimento Livre e Esclarecido.

Para inclusão do paciente nesta pesquisa, deveriam ser obedecidos os seguintes critérios de inclusão: estar em inatividade física há pelo menos seis meses, previamente ao início do estudo e ter liberação médica para realizar atividade física. Caso o paciente atendesse tais critérios, mas apresentasse alguma doença do sistema muscular, articular, ósseo e/ou do tecido conjuntivo, identificada na aplicação inicial do questionário autoadministrado, ele seria excluído do estudo.

Dessa forma, os pacientes incluídos na pesquisa foram aleatoriamente distribuídos no grupo experimental (exercício aeróbio intradialítico) e controle (sem exercício). Utilizou-se a aleatorização simples, que foi caracterizada pela entrada dos pacientes numa sala reservada dentro da clínica de doenças renais - Natal/RN, local onde o estudo foi conduzido. Nesta sala, o coordenador da pesquisa esperava o paciente com um fichário posto sobre a mesa da sala. Neste fichário, continham envelopes de cores idênticas e distribuídos aleatoriamente. $\mathrm{O}$ coordenador solicitou ao paciente que retirasse apenas 
um envelope de dentro do fichário. Dentro da cada envelope, havia a informação sobre qual grupo o paciente seria alocado. Após este procedimento, o paciente devolvia o envelope para dentro do fichário, e o seguinte repetia o mesmo procedimento.

Após determinação de quais pacientes iriam participar do estudo, eles foram submetidos às seguintes avaliaçóes.

\section{Agilidade e equilíbrio dinâmico}

A avaliação da agilidade/equilíbrio dinâmico foi conduzida de acordo com Rikli e Jones'. Nesta avaliação, os equipamentos utilizados foram cronômetro, fita métrica, cone e cadeira de encosto reto sem braços com altura de $43 \mathrm{~cm}$. Esta cadeira foi posicionada contra a parede e, na sua frente, foi colocado um cone localizado a 2,44 metros de distância, marcados desde o ponto abaixo da borda frontal da cadeira até a parte de trás do cone. $\mathrm{O}$ paciente começou o teste sentado na cadeira em posição ereta, com as mãos sobre a coxa e os pés apoiados ao solo. Ao sinal de "vai" que foi dado pelo avaliador, o paciente caminhou o mais rápido possível em direçáo ao cone, contornando-o e retornando à cadeira, devendo voltar a sua posiçáo inicial sentado. $\mathrm{O}$ avaliador iniciou a cronometragem do tempo, no momento do sinal dado e parou após o paciente ter concluído o percurso e sentado na cadeira. O paciente teve duas tentativas, separadas por intervalo de 3 minutos, e seu menor tempo para realizar o teste foi considerado.

Além desta avaliação, os pacientes eram normalmente submetidos a exames bioquímicos (Ureia $[\mathrm{mg} / \mathrm{d}$ ], Creatinina $[\mathrm{mg} / \mathrm{dl}]$, Hemácias $\left[\mathrm{mm}^{3}\right]$, Hematócrito [\%]) e fisiológicos (Pressão arterial sistólica $[\mathrm{mmHg}]$, Pressão arterial diastólica $[\mathrm{mmHg}]$ ) numa periodicidade trimestral, os quais eram realizados pelos profissionais da clínica de doenças renais. Sendo assim, o protocolo de treinamento foi caracterizado pelos seguintes procedimentos:

\section{Protocolo de treinamento aeróbio intradialítico}

Previamente ao início do programa de treinamento, os pacientes tiveram as suas frequências cardíacas de repouso coletadas, a fim de utilizar este parâmetro para calcular a intensidade do treinamento por meio do método da frequência cardíaca de reserva ${ }^{10}$. Vale salientar que foi realizada uma estimativa da frequência cardíaca máxima dos pacientes pela fórmula 220 -idade.

Neste sentido, o programa de exercício aeróbio foi realizado durante as seçốes de hemodiálise (HD), três vezes por semana, em dias alternados, durante doze semanas, sempre dentro das duas primeiras horas da seção de filtragem sanguínea dos pacientes. As sessóes de treinamento foram realizadas numa bicicleta ergométrica estacionária da marca Kikos, modelo KV9-5, num intervalo de intensidade entre $50 \%$ e $60 \%$ da frequência cardíaca de reserva. As duraçóes iniciais das sessóes de treinamento foram de 20 minutos e, a cada três semanas, eram aumentados cinco minutos na duração da sessão de exercício, de maneira que nas três últimas semanas as sessôes de treino tiveram uma duração de 35 minutos. A intensidade se manteve constante durante todo o programa de treinamento e a zona de frequência cardíaca alvo era monitorada a cada 5 minutos, junto com a escala de Borg, adaptada para cicloergômetro ${ }^{11}$.

\section{Análise estatística}

Os dados foram digitados originalmente no banco de dados do software SPSS versão 20.0 para Windows. Após a análise descritiva dos dados, foi realizado o teste de normalidade nas variáveis coletadas, seguindo os seguintes critérios de normalidade: $100 \%$ dos valores devem estar entre a média e três vezes o desvio padrão para cima e para baixo; a distribuição deve ser simétrica e, em caso de assimetria, o valor absoluto deverá ser menor do que duas vezes o seu erro padrão; o valor da Kurtose deve ser menor do que duas vezes o seu erro padrão ${ }^{12}$.

Em função das variáveis não atenderem aos critérios de normalidade, foi realizada a comparação delas dentro do mesmo grupo pelo teste de Wilcoxon. No entanto, a comparação entre os grupos foi feita pelo teste $U$ de Mann-Whitney. Para todas as análises, foi considerado um nível de significância menor que 5\%.

\section{Resultados}

Foram avaliados 34 sujeitos de ambos os gêneros, sendo $23(67,6 \%)$ do gênero masculino e $11(32,4 \%)$ do gênero feminino. No tocante ao grupo experimental, dez $(58,8 \%)$ sujeitos eram do gênero masculino e sete $(41,2 \%)$ do gênero feminino. No entanto, no grupo controle $13(76,5 \%)$ eram do gênero masculino e quatro $(23,5 \%)$ do gênero feminino. Todos os indivíduos apresentaram uma frequência de $100 \%$ ao longo do programa de treinamento, sendo, dessa forma, avaliados todos os participantes.

Com relaçáo às características no período pré-intervenção do grupo experimental e controle, podem-se observar na tabela 1 os resultados encontrados. 
Conforme a tabela 1 , percebe-se que, previamente ao início do protocolo de treinamento, ambos os grupos foram estatisticamente iguais nas variáveis analisadas. Entretanto, a creatinina foi a única variável que teve uma tendência, no limiar de significância, de ser maior no grupo controle em relação ao grupo experimental. De qualquer forma, a semelhança entre as características dos grupos no período pré-intervenção permite que a comparaçáo entre os grupos seja realizada de maneira adequada, pois ambos iniciaram o estudo apresentando as mesmas condições.

Dentro desta análise descritiva no período pré--intervenção, constata-se que os dados bioquímicos (ureia, creatinina, hemácias e o hematócrito) correspondiam aos valores normais para este tipo de público ${ }^{13}$. Com relação aos valores de pressão arterial sistólica e diastólica, pode-se sugerir que os pacientes deste estudo se trataram de pacientes compensados, pois, geralmente, este público apresenta elevados níveis de pressão arterial ${ }^{7}$.

No tocante às alteraçóes alcançadas pelo grupo experimental após as 12 semanas de intervenção, podem ser visualizados na tabela 2 os resultados encontrados.

Ao analisar as variáveis bioquímicas, constata-se que a ureia foi a única que obteve uma diminuição significativa após o período de treinamento. Já com relação às variáveis fisiológicas, observa-se que a pressão arterial sistólica apresentou, no período pós-intervenção, um intervalo de menores valores de pressão em comparação com o período pré-intervenção. Ainda dentro das variáveis fisiológicas, percebe-se que a frequência cardíaca pós-treinamento foi significativamente reduzida.

Tabela 1: Perfil pré-intervenção das medidas de tendência central e dispersão, relacionadas às variáveis antropométricas, bioquímicas, fisiológicas e funcionais nos pacientes submetidos ao tratamento de hemodiálise

\begin{tabular}{lccccc}
\hline \multicolumn{2}{c}{ Experimental } & Controle & & \\
& Mediana & $\mathbf{Q}_{25}-\mathbf{Q}_{75}$ & Mediana & $\mathbf{Q}_{25}-\mathbf{Q}_{75}$ & $\mathbf{p}$ \\
\hline Idade & 45 & $28,5-60,0$ & 47 & $26,5-57,0$ & 0,760 \\
IMC & 22,1 & $20,1-24,1$ & 22,2 & $19,4-26,5$ & 0,892 \\
Creatinina (mg/dl) & 13,1 & $9,5-14,5$ & 14,3 & $13,8-16,3$ & 0,062 \\
Hemácias (milhóes/mm $\left.\mathrm{mm}^{3}\right)$ & 3,62 & $3,01-4,32$ & 4,16 & $3,54-4,61$ & 0,140 \\
Hematócrito (\%) & 33,6 & $30,5-38,0$ & 35,0 & $32,0-38,0$ & 0,658 \\
PAS (mmHg) & 130 & $130-150$ & 140 & $120-145$ & 0,658 \\
PAD (mmHg) & 80 & $80-85$ & 80 & $80-90$ & 0,394 \\
FCR (bpm) & 84 & $69-92$ & 76 & $66-82$ & 0,193 \\
AG/ED (segundos) & 5,7 & $4,8-7,4$ & 0,322 & $5,5-7,6$ & 0,322 \\
\hline
\end{tabular}

IMC - índice de massa corporal, PAS - pressão arterial sistólica, PAD - pressão arterial diastólica, FCR - frequência cardíaca de repouso, AG/ED - agilidade/ equilíbrio dinâmico.

Tabela 2: Comparações nas variáveis bioquímicas, fisiológicas e funcionais no grupo experimental

\begin{tabular}{lccccc} 
& \multicolumn{2}{c}{ Grupo experimental pré-intervençáo } & \multicolumn{2}{c}{ Grupo experimental pós-intervençáo } \\
\cline { 2 - 6 } & Mediana & $\mathbf{Q}_{25}-\mathbf{Q}_{75}$ & Mediana & $\mathbf{Q}_{25}-\mathbf{Q}_{75}$ & $\mathbf{p}$ \\
\hline Ureia (mg/dl) & 172 & $155-206$ & $144^{\text {a }}$ & $115-190$ & 0,008 \\
Creatinina (mg/dl) & 13,1 & $9,5-14,5$ & 10 & $9,2-15,5$ & 0,570 \\
Hemácias (milhóes/mm $)$ & 3,62 & $3,01-4,32$ & 3,86 & $3,33-4,15$ & 0,687 \\
Hematócrito (\%) & 33,6 & $30,5-38,0$ & 34,8 & $30,0-37,3$ & 0,758 \\
PAS (mmHg) & 130 & $130-150$ & $130^{\text {a }}$ & $120-140$ & 0,044 \\
PAD (mmHg) & 80 & $80-85$ & 80 & $80-80$ & 0,796 \\
FCR (bpm) & 84 & $69-92$ & $71^{\text {a }}$ & $64-80$ & 0,002 \\
AG/ED (segundos) & 5,7 & $4,8-7,4$ & $5,1^{\text {a }}$ & $4,2-5,8$ & 0,0001 \\
\hline
\end{tabular}

IMC - índice de massa corporal, PAS - pressão arterial sistólica, PAD - pressão arterial diastólica, FCR - frequência cardíaca de repouso, AG/ED - agilidade/ equilíbrio dinâmico.

${ }^{a}$ Diferença significativa em relação ao período pré-intervenção $(p<0,05)$. 
O grupo experimental também teve uma redução muito significativa no tempo de execução no teste de agilidade/equilíbrio dinâmico, o que indica uma boa evolução neste aspecto.

No entanto, a fim de verificar a influência do treinamento aeróbio nos parâmetros analisados, procedeu-se com a comparação entre os grupos no período pós-intervenção, conforme pode ser visualizado na tabela 3 .

Observa-se que a ureia teve uma tendência, no limiar de significância, de ser menor no grupo experimental em relação ao controle, podendo-se sugerir uma possível influência do treinamento aeróbio na melhora desta variável. Provavelmente, esta diferença não foi significativamente estatística, em função do pequeno tamanho amostral dos grupos em questão. Em relação à pressão arterial sistólica e diastólica, o grupo experimental apresentou menores valores do que o grupo controle. Provavelmente, a diferença encontrada na pressão arterial diastólica pode ter sido em função de o grupo controle ter apresentado um aumento no período de 12 semanas do estudo. Tal sugestão foi levantada em função de o grupo experimental não ter apresentado alteraçóes desta variável no mesmo período investigado (tabela 2).

Dentro das variáveis fisiológicas, destaca-se o comportamento da frequência cardíaca, na qual se observou diminuiçáo significativa no período pós-intervenção dentro do grupo experimental (tabela 2). Entretanto, o grupo controle também teve uma alteração na faixa de valores referentes ao intervalo interquartílico após as 12 semanas do estudo, o que resultou em igualdade desta variável na comparaçáo entre os grupos.

No tocante à característica funcional dos grupos, de fato, o treinamento aeróbio foi altamente benéfico nesta variável. Esta observação justifica-se pelo fato de o grupo experimental ter cumprido a tarefa que envolvia

Tabela 3: Perfil pós-intervenção das medidas de tendência central e dispersão, relacionadas às variáveis antropométricas, bioquímicas, fisiológicas e funcionais nos pacientes submetidos ao tratamento de hemodiálise

\begin{tabular}{|c|c|c|c|c|c|}
\hline & \multicolumn{2}{|c|}{ Experimental } & \multicolumn{3}{|c|}{ Controle } \\
\hline & Mediana & $\mathbf{Q}_{25}-\mathbf{Q}_{75}$ & Mediana & $\mathbf{Q}_{25}-\mathbf{Q}_{75}$ & $p$ \\
\hline Ureia $(\mathrm{mg} / \mathrm{dl})$ & 144 & $115-190$ & 190 & $134-212$ & 0,092 \\
\hline Creatinina (mg/dl) & 10 & $9,2-15,5$ & 13 & $8,4-15,4$ & 0,865 \\
\hline Hemácias (milhões $/ \mathrm{mm}^{3}$ ) & 3,86 & $3,33-4,15$ & 3,86 & $3,36-4,28$ & 0,919 \\
\hline Hematócrito (\%) & 34,8 & $30,0-37,3$ & 35,1 & $30,3-37,0$ & 0,786 \\
\hline PAS (mmHg) & 130 & $120-140$ & $140^{\mathrm{b}}$ & $140-160$ & 0,011 \\
\hline PAD (mmHg) & 80 & $80-80$ & $90^{\mathrm{b}}$ & $85-100$ & 0,001 \\
\hline FCR (bpm) & 71 & $64-80$ & 80 & $68,5-88,0$ & 0,106 \\
\hline AG/ED (segundos) & 5,1 & $4,2-5,8$ & $6,4^{\mathrm{b}}$ & $5,8-7,5$ & 0,001 \\
\hline
\end{tabular}

IMC - índice de massa corporal, PAS - pressão arterial sistólica, PAD - pressão arterial diastólica, FCR - frequência cardíaca de repouso, AG/ED - agilidade/ equilíbrio dinâmico.

${ }^{\mathrm{b}}$ Diferença significativa em relação ao grupo experimental $(\mathrm{p}<0,05)$.

agilidade e equilíbrio dinâmico, num tempo bem menor em relação ao grupo controle. Tal diferença não existia no período pré-intervenção.

\section{Discussão}

De acordo com o que foi apresentado nos resultados, os pacientes que foram submetidos a 12 semanas de treinamento aeróbio intradialítico obtiveram uma melhora nos níveis séricos de ureia, além de uma reduçáo significativa da pressão arterial, especialmente, da pressão arterial sistólica. Adicionalmente, destacou-se o ganho funcional destes pacientes, os quais alcançaram uma redução significativa no tempo de execução no teste de agilidade/equilíbrio dinâmico.

$\mathrm{Na}$ insuficiência renal crônica, a depuração renal, ou seja, a filtração glomerular está reduzida ${ }^{14}$. Entre os indicadores da função renal, estão os valores séricos de ureia e creatinina ${ }^{15}$. Segundo Daugirdas ${ }^{16}$, a ureia é um indicador do estado de hidratação e pode quantificar a ingestão de proteínas. Neste sentido, Riella e Martins ${ }^{13}$ relataram que valores séricos de ureia acima de $200 \mathrm{mg} /$ $\mathrm{dl}$ são considerados tóxicos, especialmente, para pacientes dialíticos.

Dentro deste contexto, há evidências de que o exercício aeróbio realizado durante a HD pode gerar melhora na eficácia da diálise, a qual é aferida não só pela 
URR (remoção em percentual de ureia após a hemodiálise), como também pelo CRR (remoção em percentual de creatinina após a hemodiálise) e pelo Kt/V (representa a remoção dos solutos de uma forma geral) ${ }^{7}$.

Dessa forma, Kong et al. ${ }^{17}$ submeteram 11 pacientes ao exercício aeróbio durante a HD e avaliaram seu efeito na eficiência da diálise. Os resultados indicaram que o Kt/V apresentou uma melhora significativa. Adicionalmente, Parsons et al. ${ }^{18}$ observaram que a realização de exercício por 15 minutos dentro das três primeiras horas da HD foi capaz de melhorar o URR.

De acordo com Giannaki et al. ${ }^{7}$, um dos mecanismos que poderiam explicar a melhora na remoção da ureia durante a realização de exercício intradialítico vem pelo conhecimento de que grandes quantidades de ureia são absorvidas e armazenadas em tecidos de baixa perfusão, tais como musculatura esquelética, pele e ossos. Por outro lado, o exercício induz a uma vasodilatação e aumenta o fluxo sanguíneo para musculatura, portanto, melhora a perfusão entre fibras musculares e capilares. $\mathrm{O}$ aumento da perfusão induzida pelo exercício leva a um aumento na troca entre compartimentos intercelulares, nas musculaturas periféricas, e intravasculares. Desta forma, o aumento do fluxo sanguíneo gerado pelo exercício mobiliza a ureia intramuscular, transferindo-a para a circulaçáo sistêmica e a partir daí para o filtro da hemodiálise fora do corpo do paciente.

Todo este processo pode explicar a redução significativa que os pacientes submetidos ao exercício intradialítico deste estudo tiveram nos níveis de sérios de ureia, após as 12 semanas de intervenção. Tal estímulo não foi aplicado aos pacientes do grupo controle, os quais permaneceram com valores mais elevados.

No tocante aos aspectos fisiológicos, observou-se, neste estudo, que os pacientes do grupo experimental apresentaram uma significativa redução da pressão arterial. Corroborando com este resultado, Reboredo et al. ${ }^{1}$ submeteram um grupo de 14 pacientes à monitorização ambulatorial da pressão arterial com o objetivo de avaliar o efeito do treinamento aeróbio, realizado durante a $\mathrm{HD}$, no controle da pressão arterial. Os resultados mostraram reduçáo significativa da pressão arterial sistólica e diastólica, após 12 semanas intervenção.

Segundo Deligiannis et al. ${ }^{19}$, a melhora nos valores de pressão arterial nos pacientes submetidos à hemodiálise pode ser explicada pelo aumento, em repouso, do tônus vagal cardíaco proporcionado pela prática regular do exercício, como também em função da diminuição do estímulo simpático no vaso. Adicionalmente, os mesmos autores relataram que a redução da frequência cardíaca após execução do treinamento aeróbio intradialítico pode estar associada a um maior volume sistólico (VS) durante o repouso, e também a um aumento da densidade capilar dos músculos envolvidos na prática do exercício. Estes mecanismos podem explicar a redução da FC observada no grupo experimental dos pacientes da presente pesquisa.

Além das alteraçôes em variáveis bioquímicas e fisiológicas, sabe-se que, na DRC, a baixa capacidade funcional para atividades diárias é resultado de um conjunto de fatores, tais como alteraçóes cardiopulmonares, musculoesqueléticas, neurológicas, hidroeletrolíticas e endocrinometabólicas, as quais induzem a dispneia, síndrome urêmica, fadiga, dor em membros inferiores e fraqueza muscular generalizada ${ }^{20}$.

Adicionalmente, Medeiros et al. ${ }^{21}$ relataram que pacientes com DRC submetidos a tratamento hemodialítico sofrem atrofia muscular, uma vez que ocorrem alteraçôes importantes no sistema muscular, tendo, como consequência disso, a fraqueza generalizada de todo o organismo. Além do mais, um dos fatores que prejudica a capacidade funcional dos pacientes com DRC é que, quando um indivíduo apresenta o diagnóstico da doença, o sujeito costuma ficar por um longo período de tempo em inatividade física, levando-o ao descondicionamento físico progressivo. Dessa forma, o paciente acaba tendo limitada a sua capacidade de realizar atividades físicas e também atividades da vida diária ${ }^{22}$.

Dentro deste contexto, Martins e Cesarino ${ }^{23}$ ressaltam que a parcial impossibilidade de locomoçáo ou a dificuldade na marcha, devido ao estado clínico geral de quem possui DRC, é uma importante característica que compromete a independência funcional do paciente.

Neste sentido, Storer ${ }^{24}$ destaca que o mesmo efeito que o treinamento aeróbio pode causar em indivíduos sadios, no tocante à melhora de tarefas, tais como caminhar ou subir e descer escadas, também pode beneficiar os indivíduos com DRC. Este estudo confirma tal hipótese pela evidência observada nos resultados da tabela 3. Dessa forma, o exercício aeróbio realizado durante a sessão de hemodiálise pode aumentar a agilidade, a coordenação e o equilíbrio. Diante disso, pode-se sugerir que tais modificaçóes levem a uma redução do medo de se movimentar e a um aumento do controle motor, ocasionando, portanto, uma melhora da locomoção dos pacientes submetidos à hemodiálise.

\section{Conclusão}

Neste estudo foi observado que a realização de exercícios aeróbios durante a sessão de hemodiálise proporciona aos pacientes uma melhora nos níveis séricos de ureia, além da redução da pressão arterial. Adicionalmente, foi observado que os pacientes submetidos a este tipo de treinamento melhoram a sua capacidade funcional.

Entretanto, este estudo foi realizado com pacientes dialíticos relativamente saudáveis, os seja, pacientes que 
apresentaram boas condiçôes bioquímicas e fisiológicas. Aliado a isso, destaca-se o fato de este estudo ter tido um pequeno tamanho amostral. Sendo assim, os efeitos benéficos do exercício intradialítico, observados neste estudo, náo podem ser extrapolados para todos os pacientes com doença renal em estágio terminal, pois a maioria apresenta condiçôes diferentes dos pacientes investigados nesta pesquisa.

Em resumo, a aplicaçáo extensiva de programas de exercício nesta população ainda não foi completamente estabelecida, necessitando, assim, de mais estudos, os quais incluam pacientes com condiçóes mais desfavoráveis.

\section{Referências}

1. Reboredo MM, Henrique DMN, Chaoubah A, Paula RB. Treinamento aeróbico melhora a capacidade funcional de pacientes em hemodiálise crônica. Arq Bras Cardiologia. 2010 Jan; 94(6):823-8.

2. Romão Júnior JE. Insuficiência renal crônica. In: Cruz J, Praxedes JN, Cruz HMM. Nefrologia. 2th ed. São Paulo: Sarvier; 2006.

3. Parsons TL, Toffelmire EB, King-Vanvlack CE. The effect of an exercise program during hemodialysis on dialysis efficacy, blood pressure and quality of life in endstage renal disease (ESRD) patients. Clin Nephrol. 2004 Apr; 61(4):261-74.

4. Monteiro MF, Sobral Filho DC. Exercício físico e o controle da pressão arterial. Rev Bras Med Esporte. 2004 Nov-Dez; 10(6):513-6.

5. Moura RMF, Silva FCR, Ribeiro GM, Sousa LA. Efeitos do exercício físico durante a hemodiálise em indivíduos com insuficiência renal crônica: uma revisão. Fisioterapia e Pesquisa. 2008 Jan; 15(1):86-91.

6. Konstantinidou E, Koukouvou G, Kouidi E, Deligiannis A, Tourkantonis A. Exercise training in patients with endstage renal disease on hemodialysis: comparison of three rehabilitation programs. J Rehabil Med. 2002 Jan; 34(1):40-5.

7. Giannaki CD, Stefanidis I, Karatzaferi C, Liakos N, Roka V, Ntente I, et al. The Effect of Prolonged Intradialytic Exercise in Hemodialysis Efficiency Indices. ASAIO J. 2011 May-Jun; 57(3): 213-8.

8. Cheema BS, Singh MA. Exercise training in patients receiving maintenance hemodialysis: A systematic review of clinical trials. Am J Nephrol. 2005 Jul-Aug; 25(4): 352-64.

9. Rikli RE, Jones CJ. Developtment and validation of a functional fitness test for community-residing older adults. J Aging Phys Act. 1999 Mar; 7, 129-161.

10. Karvonen MJ, Kentala E, Mustala O. The effects of training on heart rate; a longitudinal study. Ann. Med. Exp. Biol. Fenn. 1957; 35(3): 307-15.

11. Robertson RJ, Goss FL, Dube J, Rutkowski J, Dupain M, Brennan C, et al. Validation of the Adult OMNI Scale of Perceived Exertion for Cycle Ergometer Exercise. Med Sci Sports Exerc. 2004 Jan; 36(1): 102-8.

12. Martinéz-González A, Villegas AS, Fajardo JF. Bioestadística Amigable. 1th ed. Espanha: Diaz de Santos; 2009.

13. Riella CM, Martins C. Nutriçáo e o Rim. 1th ed. Rio de Janeiro: Guanabara Koogan; 2001.

14. Riella, CM. Princípios de nefrologia e distúrbios hidroeletrolíticos. 2th ed. Rio de Janeiro: Guanabara Koogan; 2003.

15. Depner T, Himmelfarb J. Uremic retention solutes: The free and the bound. J Am Soc Nephrol. 2007 Mar; 18(3): 675-6.

16. Daugirdas JT. Second generation logarithmic estimates of single-pool variable volume Kt/V: An analysis of error. J Am Soc Nephro. 1993 Nov; 4(5): 1205-13.

17. Kong CH, Tattersall JE, Greenwood RN, Farrington K. The effect of exercise during haemodialysis on solute removal. Nephrol Dial Transplant. 1999 Dec;14(12): 2927-31.

18. Parsons TL, Toffelmire EB, King-VanVlack CE. The effect of an exercise program during hemodialysis on dialysis efficacy, blood pressure and quality of life in end-stage renal disease (ESRD) patients. Clin Nephrol. 2004 Apr; 61(4): 261-74.

19. Deligiannis A, Kouidi E, Tassoulas E, Gigis P, Tourkantonis A, Coats A. Cardiac effects of exercise rehabilitation in hemodialysis patients. Int J Cardiol. 1999 Aug; 70(3): 253-66.

20. Bronas UG. Exercise training and reduction of cardiovascular disease risk factors in patients with chronic kidney disease. Adv Chronic Kidney Dis. 2009 Nov; 16(6): 449-58.

21. Medeiros RH, Pinent CE, Meyer F. Aptidão física de individuo com doença renal crônica. J Bras Nefrol. 2002 Jun; 24(2): 81-7.

22. Painter P. Physical functioning in end-stage renal disease patients: Update 2005. Hemodial Int. 2005 Jul; 9(3): 218-35.

23. Martins MRI, Cesarino CB. Qualidade de vida de pessoas com doença renal crônica em tratamento hemodialítico. Rev Latino-am Enfermagem. 2005 Set-Out; 13 (5): 670-6.

24. Storer TW, Casaburi R, Sawelson S, Kopple JD. Endurance exercise training during haemodialysis improves strength, power, fatigability and physical performance in maintenance haemodialysis patients. Nephrol Dial Transplant. 2005 Jul; 20(7): 1429-37. 